\title{
An Improved Algorithm for the Metro-line Crossing Minimization Problem
}

\author{
Martin Nöllenburg \\ Fakultät für Informatik, Universität Karlsruhe $(\mathrm{TH})$ and \\ Karlsruhe Institute of Technology (KIT), Karlsruhe, Germany \\ noellenburg@iti.uka.de
}

\begin{abstract}
In the metro-line crossing minimization problem, we are given a plane graph $G=(V, E)$ and a set $\mathcal{L}$ of simple paths (or lines) that cover $G$, that is, every edge $e \in E$ belongs to at least one path in $\mathcal{L}$. The problem is to draw all paths in $\mathcal{L}$ along the edges of $G$ such that the number of crossings between paths is minimized. This crossing minimization problem arises, for example, when drawing metro maps, in which multiple transport lines share parts of their routes.

We present a new line-layout algorithm with $O\left(|\mathcal{L}|^{2} \cdot|V|\right)$ running time that improves the best previous algorithms for two variants of the metro-line crossing minimization problem in unrestricted plane graphs. For the first variant, in which the so-called periphery condition holds and terminus side assignments are given in the input, Asquith et al. 1 gave an $O\left(|\mathcal{L}|^{3} \cdot|E|^{2.5}\right)$-time algorithm. For the second variant, in which all lines are paths between degree-1 vertices of $G$, Argyriou et al. 22 gave an $O\left(\left(|E|+|\mathcal{L}|^{2}\right) \cdot|E|\right)$-time algorithm.
\end{abstract}

\section{Introduction}

Schematic metro maps are effective and popular visualizations of public transport networks all over the world; see Ovenden's comprehensive collection of metro maps [3. Several methods for automatically drawing metro maps have been suggested in recent years [4, 5, 6. These methods, however, focus on drawing the underlying graph, that is, the graph that represents stations as vertices and direct links between two stations as edges. This graph represents the infrastructure of the transport network, for example, railway tracks or roads. A schematic layout of the underlying graph, whether created manually or by one of the existing methods mentioned above, does not necessarily yield a proper metro map yet. The reason is that most real-world networks contain many different transport lines whose routes partially overlap, that is, some edges of the underlying graph are shared by multiple transport lines. In practice, each transport line is therefore drawn in a distinct color along the edges of its path in the underlying graph. Consequently, edges that belong to several lines consist in fact of a bundle of colored parallel curves. As an example, Fig. 1] shows a detail of the metro map of Cologne. 


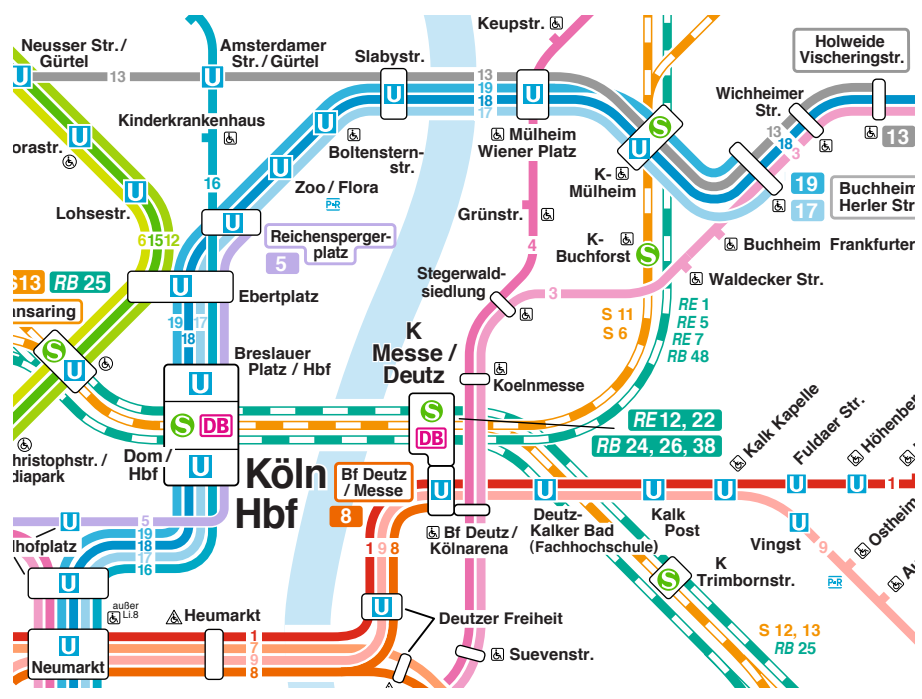

Fig. 1. Detail of the metro map of Cologne

An immediate consequence of such a visualization is that there are situations in which two lines in the - otherwise plane - network cross. Some line crossings are mandatory, induced by the prescribed network topology, others depend on the line orders in each vertex and can be avoided by choosing the right orders. Hence, the metro-line crossing minimization (MLCM) problem arises as a secondary problem in the metro-map layout process: find an ordering of the parallel lines along each edge of the underlying graph such that as few pairs of lines as possible cross each other in the final layout. Additionally, the relative order of lines traversing a vertex in the same direction must not change within this vertex, that is, we do not allow to hide line crossings "below" the area occupied by the representation of a vertex. Note that the MLCM problem is independent of the actual layout of the underlying graph. The combinatorial embedding of the underlying graph, which is usually defined by its geographic input embedding, is all one needs to define the orderings of the parallel lines. Hence, algorithms for MLCM can be used both for reducing line crossings in existing layouts and, as a second step in combination with layout methods for the underlying graph, for creating metro maps from scratch.

Although we present our results in terms of the classic problem of visualizing transportation networks, we note that the metro map metaphor has also been used as a means to visualize potentially much larger networks in other fields, for example, metabolic pathways [7]. Actually, the MLCM problem appears whenever multiple parallel edges in a graph need to be drawn separately along a common geometric path with the minimum number of crossings among them.

Benkert et al. 8] introduced the general MLCM problem. Subsequently, MLCM was considered in several variants and for different classes of underlying graphs [9, 2, 1, which are discussed in detail in Section 3. One important 


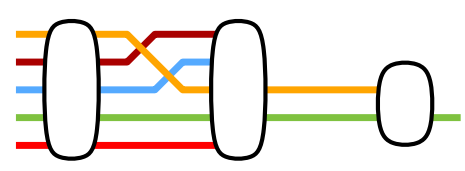

(a) With periphery condition.

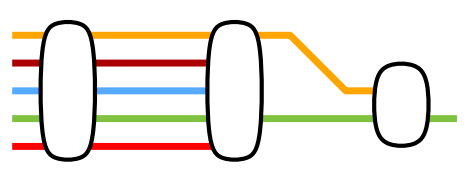

(b) Without periphery condition.

Fig. 2. Layout of a terminus (middle vertex) with three terminating lines. The layout in (b) introduces a gap between the continuing lines.

variant, posed as an open problem by Benkert et al. [8] and addressed by Bekos et al. 9] and by Asquith et al. 1, restricts the positions of each line's start and end point (called termini) to be left- or rightmost in the ordered sequence of lines along the underlying edges leading to its termini. This restriction is called the periphery condition and prevents gaps between continuing lines, see Fig. 2 Gaps between parallel lines disrupt the uniform appearance of the underlying edge and hence are to be avoided in order to improve readability. Apart from avoiding gaps, an exposed outer position for terminating lines also allows for better highlighting or labeling of the termini. Often the final destination of a train or bus in a transport network is used to indicate its direction and hence termini and their names should be prominent features that are easy to recognize in a metro map. Many of the real-world maps in Ovenden's collection [3] adhere to the periphery condition, as does the metro map of Cologne in Fig. 1] Bekos et al. 9] showed that the MLCM problem is NP-hard under the periphery condition if each terminus can lie on either side of the respective final edge. On the other hand, Asquith et al. 1] showed that the MLCM problem under the periphery condition can be solved efficiently for general plane graphs if the terminus side assignment is given as part of the input.

In this paper, we investigate the MLCM problem under the periphery condition with terminus side assignments and present a new algorithm in Section 4 that solves this problem in $O\left(|\mathcal{L}|^{2} \cdot|V|\right)$ time for a graph $G=(V, E)$ and a set of lines $\mathcal{L}$. The algorithm has two phases. First, for each pair of lines that share a common subpath, we determine their required relative order at the end of their common subpath. Then, in a second step, we iteratively insert one line at a time into the layout such that the relative orders computed in the first phase are respected and no unnecessary line crossings are created. Our algorithm improves the algorithm of Asquith et al. 11 for the same problem, which has a running time of $O\left(|\mathcal{L}|^{3} \cdot|E|^{2.5}\right)$. Our algorithm can also be used to solve a closely related problem considered by Argyriou et al. [2], where all lines must be paths connecting two degree-1 vertices in $G$. Hence, it also improves the algorithm of Argyriou et al., which has a running time of $O\left(\left(|E|+|\mathcal{L}|^{2}\right) \cdot|E|\right)$. These are the only two variants of MLCM that are known to be efficiently solvable, and our algorithm is to the best of our knowledge currently the fastest method to solve both of them for general plane underlying graphs. 


\section{Model}

The input to the MLCM problem is a metro graph $(G, \mathcal{L})$, where $G=(V, E)$ is a planar embedded graph and $\mathcal{L}$ is a line cover of $G$, that is, a set of simple paths (or lines) that cover $G$. Note that existing edge crossings in the input graph can easily be modeled as dummy vertices. For notational convenience, we consider each undirected edge $\{u, v\} \in E$ as a pair of directed edges $u v$ and $v u$. Both notations refer to the same single edge just from two different perspectives.

The vertices $v_{0}$ and $v_{k}$ of a line $\ell=\left(v_{0}, v_{1}, \ldots, v_{k}\right) \in \mathcal{L}$ of length $|\ell|=k$ are called the termini of $\ell$, the vertices $v_{1}, \ldots, v_{k-1}$ are called intermediate vertices of $\ell$. An edge $u v$ is included in a line $\ell$, in short $u v \in \ell$, if $u$ and $v$ are consecutive vertices in $\ell$. We denote as $\mathcal{L}_{u v}=\mathcal{L}_{v u}=\{\ell \in \mathcal{L} \mid u v \in \ell\}$ the set of all lines that include an edge $u v$. The total edge size of $\mathcal{L}$ is defined as $N_{\mathcal{L}}=\sum_{\ell \in \mathcal{L}}|\ell|=$ $\sum_{u v \in E}\left|\mathcal{L}_{u v}\right|$. Note that $N_{\mathcal{L}} \in O(|\mathcal{L}| \cdot|V|)$ since $|\ell| \leq|V|$ for each line $\ell \in \mathcal{L}$.

Each vertex $u$ has a cyclic sequence of $\sum_{u v \in E}\left|\mathcal{L}_{u v}\right|$ consecutive ports, one for each line of each incident edge $u v$. Each port is a point on the boundary of the geometric representation of $u$, at which the individual lines in $\bigcup_{u v \in E} \mathcal{L}_{u v}$ enter (or leave) $u$. We are interested in the order in which the lines in $\mathcal{L}_{u v}$ connect to the consecutive subsequence of ports of $u$ (and of $v$ ) that correspond to the lines along edge $u v$. So for each edge $u v \in E$, we define two line orders $<_{u v}^{u}$ and $<_{u v}^{v}$ of $\mathcal{L}_{u v}$ in the endpoints of $u v$. For two lines $\ell_{1}$ and $\ell_{2}$ in $\mathcal{L}_{u v}$ we write $\ell_{1}<_{u v}^{u} \ell_{2}$ (or $\ell_{1}<_{u v}^{v} \ell_{2}$ ) if $\ell_{1}$ is right of $\ell_{2}$ at the endpoint $u$ (or $v$ ) with respect to the direction of $u v$. Note that the orders are reversed if we use the oppositely directed edge $v u$ instead of $u v$, that is, $\ell_{1}<_{u v}^{u} \ell_{2}$ if and only if $\ell_{2}<_{v u}^{u} \ell_{1}$. The sorted sequence of the lines in $\mathcal{L}_{u v}$ with respect to $<_{u v}^{u}$ is denoted as $s_{u v}^{u}$; analogously $s_{u v}^{v}$ is the sorted sequence of lines with respect to $<_{u v}^{v}$. Again, the sequences $s_{v u}^{u}$ and $s_{v u}^{v}$ are the reversed sequences of $s_{u v}^{u}$ and $s_{u v}^{v}$.

A line crossing is a crossing between two lines $\ell_{1}$ and $\ell_{2}$ along a shared edge $u v$. The two lines cross on $u v$ if $\ell_{1}<_{u v}^{u} \ell_{2}$ and $\ell_{2}<_{u v}^{v} \ell_{1}$ or vice versa. Abstracting from geometry, the number of line crossings along an edge $u v$ is thus equal to the number of inversions in the sequences $s_{u v}^{u}$ and $s_{u v}^{v}$.

In order to avoid confusion for the map viewer, it is not allowed to hide line crossings "below" a vertex. To that end we define a line order $<_{u v}^{v}$ to be compatible with the vertex $v$ if the following holds. Apart from $u v$, let $v w_{1}, v w_{2}, \ldots, v w_{k}$ be the other edges incident to $v$ in counterclockwise order starting from $u v$. We consider the sequence $s_{u v}^{v}$ and the concatenated sequence $s^{\prime}=\prod_{i=1}^{k} s_{v w_{i}}^{v}$. Then $<_{u v}^{v}$ is compatible with $v$ if $s_{u v}^{v}$ is a subsequence of $s^{\prime}$. In other words, the lines that enter $v$ through the edge $u v$ and leave $v$ through the edges $v w_{1}, v w_{2}, \ldots, v w_{k}$ do not change their relative order. We say that a vertex $v$ is admissible if the line orders for all incident edges are compatible with $v$.

\section{MLCM Variants and Previous Work}

In this section we present four different variants of the MLCM problem that have been considered in the literature so far. Previous results and the results obtained in this paper are summarized in Table 1 
Table 1. Overview of results for the MLCM problem and its variants. Algorithmic results are given by their running time.

\begin{tabular}{|c|l|c|l|l|}
\hline problem & \multicolumn{1}{|c|}{ graph class } & restrictions & \multicolumn{1}{|c|}{ result } & reference \\
\hline \hline MLCM & single edge $u v$ & - & $O\left(\left|\mathcal{L}_{u v}\right|^{2}\right)$ & {$[8]$} \\
\hline \multirow{2}{*}{ MLCM-P } & path & - & NP-hard & {$[9]$} \\
& plane graph & - & ILP + MLCM-PA & {$[1]$} \\
\hline \multirow{3}{*}{ MLCM-PA } & path & 2-side model & $O(|\mathcal{L}| \cdot|V|)$ & {$[9]$} \\
& left-to-right tree & 2-side model & $O(|\mathcal{L}| \cdot|V|)$ & {$[9]$} \\
& plane graph & - & $O\left(|\mathcal{L}|^{3} \cdot|E|^{2.5}\right)$ & {$[1]$} \\
& plane graph & 2-side model & $O(|V| \cdot(|E|+|\mathcal{L}|))$ & {$[2]$} \\
& plane graph & - & $O\left(|\mathcal{L}|^{2} \cdot|V|\right)$ & Theorem 1 \\
\hline \multirow{5}{*}{ MLCM-T1 } & left-to-right tree & 2-side model & $O(|\mathcal{L}| \cdot|V|)$ & {$[9]$} \\
& plane graph & 2-side model & $O(|V| \cdot(|E|+|\mathcal{L}|))$ & {$[2]$} \\
& plane graph & - & $O\left(\left(|E|+|\mathcal{L}|^{2}\right) \cdot|E|\right)$ & {$[2]$} \\
& plane graph & - & $O\left(|\mathcal{L}|^{2} \cdot|V|\right)$ & Corollary 1 \\
\hline
\end{tabular}

The original metro-line crossing minimization problem as introduced by Benkert et al. 8] is as follows.

Problem 1 (MLCM). Given a metro graph $(G=(V, E), \mathcal{L})$, find for each edge $u v \in E$ two line orders $<_{u v}^{u}$ and $<_{u v}^{v}$ of the lines in $\mathcal{L}_{u v}$ such that the number of line crossings is minimal and all vertices are admissible.

A solution to MLCM is denoted as a line layout. Benkert et al. 8] gave a quadratic-time algorithm to solve MLCM for a single edge of $G$. Their algorithm does not extend to larger subgraphs and it is a remaining open problem whether MLCM is NP-hard in its general form.

We have already introduced the periphery condition, which additionally requires that each line terminates in an outer or peripheral position in each of its two termini (recall Fig. 2). Formally, this means that for each vertex $v$ and each edge $u v$ all lines in $\mathcal{L}_{u v}$, for which $v$ is a terminus, must be placed in the beginning or in the end of the sequence $s_{u v}^{v}$. In other words, no terminating line can lie between two continuing lines in the order $<_{u v}^{v}$. We denote the following variant as MLCM with periphery condition (MLCM-P).

Problem $2(M L C M-P)$. Given a metro graph $(G=(V, E), \mathcal{L})$, find for each edge $u v \in E$ two line orders $<_{u v}^{u}$ and $<_{u v}^{v}$ of the lines in $\mathcal{L}_{u v}$ such that the number of line crossings is minimal, all vertices are admissible, and each terminating line is placed at a peripheral position in each of its two termini.

Bekos et al. 9] showed that MLCM-P is NP-hard, even if $G$ is a path, and Asquith et al. 11 formulated an integer linear program (ILP) to solve MLCM-P. Still, Problem 2 gives rise to a closely related (but computationally feasible) variant that additionally specifies in the input fixed terminus sides for each line. We denote this variant as MLCM with periphery condition and terminus side assignments (MLCM-PA). 
Problem 3 (MLCM-PA). Given a metro graph $(G=(V, E), \mathcal{L})$ and terminus side assignments for all lines in $\mathcal{L}$, find for each edge $u v \in E$ two line orders $<_{u v}^{u}$ and $<_{u v}^{v}$ of the lines in $\mathcal{L}_{u v}$ such that the number of line crossings is minimal, all vertices are admissible, and each terminating line is placed at a peripheral position on the specified side of each of its two termini.

Problem 3 occurs in situations, in which, for example, the physical location of the tracks or the bus stop of the terminating line in a terminus yields this information. Alternatively, the optimal terminus side assignments can be obtained from the ILP formulation of Asquith et al. [1]. Asquith et al. also presented an $O\left(|\mathcal{L}|^{3} \cdot|E|^{2.5}\right)$-time algorithm to solve MLCM-PA for general plane graphs. Bekos et al. 9] gave two algorithms to solve MLCM-PA in the restricted 2-side model for paths and for a special class of left-to-right directed trees with bounded vertex degree in $O(|\mathcal{L}| \cdot|V|)$ time, respectively. In the 2-side model, all vertices are drawn as rectangles and all lines are drawn as $x$-monotone paths that pass through vertices from the left to the right side. Argyriou et al. [2] recently presented an algorithm to solve MLCM-PA in the 2-side model for general plane graphs in $O(|V| \cdot(|E|+|\mathcal{L}|))$ time.

Another interesting MLCM variant restricts the lines in $\mathcal{L}$ to terminate at degree-1 vertices only, that is, all termini in $(G, \mathcal{L})$ are leaves of $G$.

Problem 4 (MLCM-T1). Given a metro graph $(G=(V, E), \mathcal{L})$ in which the degree of any terminus $v$ of any path in $\mathcal{L}$ equals 1 , find for each edge $u v \in E$ two line orders $<_{u v}^{u}$ and $<_{u v}^{v}$ of the lines in $\mathcal{L}_{u v}$ such that the number of line crossings is minimal and all vertices are admissible.

Problem 4 is of practical interest since in many real-world networks transport lines lead from one terminus station in the outskirts of a city through the city center to another terminus station in the outskirts. This is exactly the situation in which lines terminate at leaves of the underlying graph. Argyriou et al. 22 presented an algorithm to solve MLCM-T1 in general plane graphs in $O\left(\left(|E|+|\mathcal{L}|^{2}\right) \cdot|E|\right)$ time. For MLCM-T1 in the previously mentioned 2-side model, they improved the running time to $O((|E|+|\mathcal{L}|) \cdot|V|)$.

We observe that a line layout for an MLCM-T1 instance trivially satisfies the periphery condition. Since each terminus $v$ is a degree- 1 vertex in $G$, there cannot be any continuing lines in $v$, and any position in the line order at $v$ is peripheral by definition. Furthermore, there is no need to distinguish two different sides for the assignment of the terminus positions: not being separated by a continuing line, the two sides of the edge leading to $v$ coincide. Hence, we can reduce any MLCM-T1 instance to an equivalent MLCM-PA instance by assigning all lines that terminate at the same leaf $v$ to the same terminus side. This actually means that there is no restriction to the line order in $v$ at all, and we indeed model the general setting of MLCM-T1. Obviously, the reduction takes only linear time. This is summarized in the following lemma.

Lemma 1. An instance of $M L C M-T 1$ can be reduced to an equivalent instance of MLCM-PA in linear time. 


\section{An Improved Algorithm for MLCM-PA}

In this section we present our main result, an $O\left(|\mathcal{L}|^{2} \cdot|V|\right)$-time algorithm for MLCM-PA and MLCM-T1 in general plane graphs. We first show a simple lemma about the line crossings in an optimal layout for an MLCM-PA instance. We define a line crossing of two lines in a metro graph $(G, \mathcal{L})$ to be unavoidable, if it is present in any line layout of $(G, \mathcal{L})$.

Lemma 2. Given a metro graph $(G, \mathcal{L})$ and terminus side assignments for all lines in $\mathcal{L}$, all line crossings in a crossing-minimal line layout are unavoidable crossings.

Proof. By definition every unavoidable crossing is present in any crossing-minimal line layout. We want to show that the opposite is also true: every line crossing in a crossing-minimal line layout is unavoidable.

So let $\ell_{1}$ and $\ell_{2}$ be two lines that cross in a crossing-minimal line layout along an edge $u v$. By $P=\left(w_{0}, \ldots, w_{i}=u, w_{i+1}=v, \ldots, w_{k}\right), 0 \leq i<k$, we denote the maximal common subpath of $\ell_{1}$ and $\ell_{2}$ that contains $u v$. First of all note that the crossing along $u v$ is the only crossing of $\ell_{1}$ and $\ell_{2}$ along $P$; any two consecutive crossings of two lines along a common subpath could be removed by routing the upper line just below the lower line along the edges between the two crossings - this contradicts the optimality of the line layout and has been observed by Asquith et al. [1 before.

We can assume that $\ell_{1}<_{u v}^{u} \ell_{2}$ and $\ell_{2}<_{u v}^{v} \ell_{1}$. Since there is a single crossing between $\ell_{1}$ and $\ell_{2}$ along $P$, this implies that $\ell_{1}<_{w_{0} w_{1}}^{w_{0}} \ell_{2}$ and $\ell_{2}<_{w_{k-1}}^{w_{k}} w_{k} \ell_{1}$. This inversion of $\ell_{1}$ and $\ell_{2}$ in the line orders of vertices $w_{0}$ and $w_{k}$ is either enforced by the combinatorial embedding of $G$ as the line orders $<_{w_{0} w_{1}}^{w_{0}}$ and $<_{w_{k-1} w_{k}}^{w_{k}}$ must be compatible with $w_{0}$ and $w_{k}$ (if the respective line continues beyond $w_{0}$ or $w_{k}$ ) or by the given terminus side assignment (if the respective line terminates at $w_{0}$ or $w_{k}$ ). The only case where the relative order of $\ell_{1}$ and $\ell_{2}$ is not fixed by the compatibility requirements or the terminus side assignments is if both lines terminate at the same vertex, say $w_{0}$, and are assigned to the same terminus side. In that case, however, they can always be reordered in $<_{w_{0} w_{1}}^{w_{0}}$ such that they reflect their relative order in $<_{w_{k-1} w_{k}}^{w_{k}}$ and the crossing would disappear. This contradicts the optimality of the layout.

We conclude that the crossing of $\ell_{1}$ and $\ell_{2}$ is unavoidable: the relative order of $\ell_{1}$ and $\ell_{2}$ at one end of $P$ is the inverse of their order at the other end of $P$ due to the given terminus side assignments or the compatibility requirements for the embedding of $G$.

Lemma 2 implies that there is a line layout that realizes exactly the unavoidable crossings and, consequently, that any such layout is optimal. Algorithm 1 constructs such a line layout. It first computes all maximal common subpaths of all pairs of lines to determine their relative orders as induced by the topology or the terminus side assignments. In a second phase all lines are iteratively inserted into the line orders of their edges and the final line layout is fixed. 


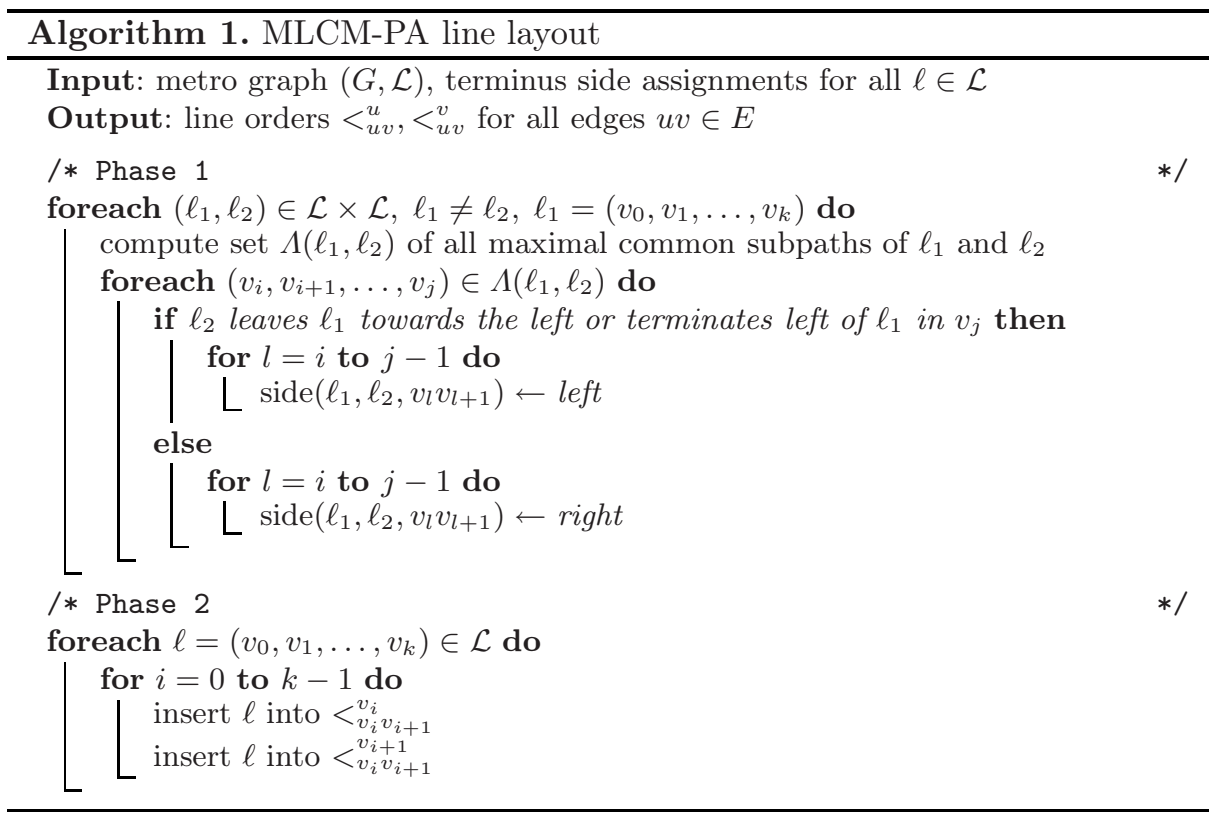

Theorem 1. Given a metro graph $(G, \mathcal{L})$ and terminus side assignments for all lines in $\mathcal{L}$, Algorithm 1 computes a crossing-minimal line layout under the periphery condition in $O\left(|\mathcal{L}| \cdot N_{\mathcal{L}}\right)$ time.

Proof. In Phase 1 of Algorithm 1 we compute the value of a binary variable side $\left(\ell_{1}, \ell_{2}, u v\right)$ for each triple of two lines $\ell_{1}$ and $\ell_{2}$ and an edge $u v$ such that $u v$ is a common edge of $\ell_{1}$ and $\ell_{2}$. This value represents the side to which line $\ell_{2}$ tends with respect to $\ell_{1}$ on edge $u v$. So if $\operatorname{side}\left(\ell_{1}, \ell_{2}, u v\right)=$ left (right), we know that at the end of the maximal common subpath of $\ell_{1}$ and $\ell_{2}$ that contains $u v$ the line $\ell_{2}$ must be placed left (right) of $\ell_{1}$.

In order to compute the set $\Lambda\left(\ell_{1}, \ell_{2}\right)$ of maximal common subpaths of $\ell_{1}$ and $\ell_{2}$ we walk along $\ell_{1}=\left(v_{0}, \ldots, v_{k}\right)$ and check for each edge $v_{i} v_{i+1}$ whether $\ell_{2}$ shares that edge with $\ell_{1}$. If this is the case, we either open a new subpath or extend the current subpath. Otherwise we close the current subpath if there is one. We assume that the input $(G, \mathcal{L})$ contains a Boolean edge-line array of size $|E| \times|\mathcal{L}|$ so that we can check whether a line uses an edge in constant time.

For each subpath $\lambda=\left(v_{i}, v_{i+1}, \ldots, v_{j}\right) \in \Lambda\left(\ell_{1}, \ell_{2}\right)$ we need to determine whether $\ell_{2}$ tends left- or rightward along $\lambda$ with respect to $\ell_{1}$, that is, whether at the end of $\lambda$ the line $\ell_{2}$ must be left or right of $\ell_{1}$. There are three cases to consider.

(1) If $v_{j}=v_{k}$, that is, $\ell_{1}$ terminates in $v_{j}$, and $\ell_{2}$ does not terminate in $v_{j}$, then $\ell_{2}$ tends leftward (rightward) if $\ell_{1}$ is assigned a right (left) terminus position, respectively. 
(2) If $v_{j}=v_{k}$ and $\ell_{2}$ also terminates in $v_{j}$, then either $\ell_{1}$ and $\ell_{2}$ are assigned to different terminus sides and $\ell_{2}$ tends to its assigned side, or both are assigned to the same side. In the latter case, $\ell_{2}$ shall stay on the same side of $\ell_{1}$ as in the first vertex $v_{i}$ of $\lambda$. So if $\ell_{2}$ enters $v_{i}$ to the left of $\ell_{1}$, then $\ell_{2}$ also tends leftward along $\lambda$; otherwise it tends rightward.

(3) If $v_{j} \neq v_{k}$ then $\ell_{2}$ tends leftward if either $\ell_{2}$ is assigned to terminate on the left in $v_{j}$ or $\ell_{2}$ continues along an edge $v_{j} w$ that is left of $\ell_{1}$ in the embedding of the underlying graph $G$; otherwise $\ell_{2}$ tends rightward.

In all three cases the value of $\operatorname{side}\left(\ell_{1}, \ell_{2}, u v\right)$ is either an immediate consequence of the lines' terminus assignments or can be determined by a constant-time query for the relative order of three incident edges in the embedding of $G$.

Summarizing the above, Phase 1 takes $O\left(|\mathcal{L}| \cdot N_{\mathcal{L}}\right)$ time and space since we check for each edge of each line if any of the other lines in $\mathcal{L}$ share the edge; if this is the case we assign the leftward/rightward value to the corresponding variable.

In Phase 2 the actual line layout is computed by iteratively fixing the course of each line. We show the correctness of the algorithm by maintaining two invariants during Phase 2.

Invariant 1: There are no invalid intra-vertex crossings, that is, for each vertex $u$ and each edge $u v$ the line order $<_{u v}^{u}$ is compatible with $u$.

Invariant 2: All line crossings are unavoidable crossings with respect to the input embedding of $G$ and the given terminus side assignments.

Inserting the first line as the only line into the empty line orders clearly satisfies both invariants. So assume that we already have a partial line layout that satisfies the invariants and that we want to insert the next line $\ell=\left(v_{0}, v_{1}, \ldots, v_{k}\right)$ into this partial layout.

We start by inserting $\ell$ into the order $<_{v_{0} v_{1}}^{v_{0}}$. Let's assume $\ell$ is assigned to a left terminus in $v_{0}$ with respect to the first edge $v_{0} v_{1}$ (for a right terminus the insertion is analogous). If $\ell$ is currently the only line with a left terminus on this edge, we insert $\ell$ as the last edge into $<_{v_{0}}^{v_{0}}$. Otherwise we scan the lines with a left terminus in $<_{v_{0} v_{1}}^{v_{0}}$, starting with the largest (or leftmost) element, for the first line $\ell^{\prime}$ for which $\operatorname{side}\left(\ell, \ell^{\prime}, v_{0} v_{1}\right)=$ right. We insert $\ell$ into $<_{v_{0}}^{v_{0}}$ immediately after (or left) of $\ell^{\prime}$. This first insertion does not create any intravertex crossings, so Invariant 1 is clearly satisfied. Furthermore, if there are multiple lines terminating along $v_{0} v_{1}$ on the same side as $\ell$ then $\ell$ is inserted exactly between those lines that tend leftward and those lines that tend rightward with respect to $\ell$. Hence all those lines are already on the correct side of $\ell$ and no line crossings are created; Invariant 2 is satisfied.

Next, we consider inserting $\ell$ into the order $<_{v_{i} v_{i+1}}^{v_{i}}$ for $i>0$ such that Invariant 1 is satisfied. If one of the neighboring lines in the previous line order $<_{v_{i-1} v_{i}}^{v_{i}}$ also continues along $v_{i} v_{i+1}$, then $\ell$ simply keeps its position directly next to that line. Since the previous layout did not contain any invalid intra-vertex crossings and $\ell$ follows a previous line, Invariant 1 is still satisfied. This case is illustrated in Figure 3a, where the red line $\ell_{1}$ follows the neighboring black line through the 


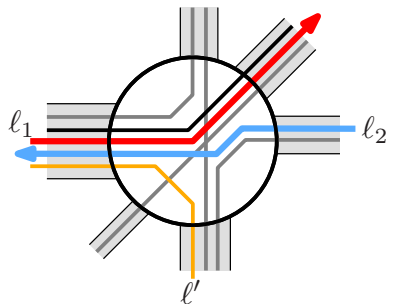

(a) Lines $\ell_{1}$ and $\ell_{2}$ are inserted so that Invariant 1 is maintained.

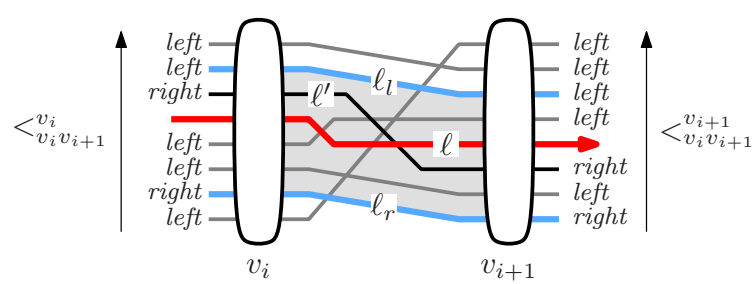

(b) Line $\ell$ is inserted into $\left\langle v_{i} v_{i+1}\right.$ so that Invariant 2 is maintained. The values $\operatorname{side}\left(\ell, \cdot, v_{i} v_{i+1}\right)$ are indicated for all lines.

Fig. 3. Insertion of lines into an existing partial line layout

vertex. Otherwise, if $\ell$ is the only line continuing along $v_{i} v_{i+1}$, we scan $<v_{i} v_{i+1}$, starting with the smallest (rightmost) element, for the first line $\ell^{\prime}$, whose previous edge $w v_{i}$ is left of $\ell$ in the embedding of $G$ or that terminates in $v_{i}$ with a left terminus along $v_{i} v_{i+1}$. We insert $\ell$ immediately before $\ell^{\prime}$ in $<_{v_{i} v_{i+1}}^{v_{i}}$. This is illustrated in Figure $3 \mathrm{a}$ by the blue line $\ell_{2}$ which is inserted immediately before the yellow line $\ell^{\prime}$. If no line $\ell^{\prime}$ is found then $\ell$ becomes the largest (leftmost) element in $<_{v_{i} v_{i+1}}^{v_{i}}$. The chosen position for $\ell$ ensures that $<_{v_{i} v_{i+1}}^{v_{i}}$ remains compatible with $v_{i}$ and that Invariant 1 is satisfied.

It remains to determine the position of $\ell$ in the order $<_{v_{i} v_{i+1}}^{v_{i+1}}$. Figure 3b illustrates the situation. We scan the already determined line order $<_{v_{i}}^{v_{i}} v_{i+1}$ for the smallest (rightmost) line $\ell_{l}$ left of $\ell$ for which $\operatorname{side}\left(\ell, \ell_{l}, v_{i} v_{i+1}\right)=$ left and for the largest (leftmost) line $\ell_{r}$ right of $\ell$, for which $\operatorname{side}\left(\ell, \ell_{r}, v_{i} v_{i+1}\right)=$ right. Note that it is possible that one or both lines $\ell_{l}$ and $\ell_{r}$ do not exist. If they exist, these two lines $\ell_{l}$ and $\ell_{r}$ are the closest lines to $\ell$ that are already on the correct side. Since Invariant 2 holds for the previous partial layout, $\ell_{l}$ and $\ell_{r}$ do not cross each other along $v_{i} v_{i+1}$, that is, $\ell_{r}<v_{i} v_{i} v_{i+1} \ell_{l}$ and $\ell_{r}<v_{i} v_{i+1} \ell_{l}$. Obviously, $\ell$ may not cross either of them and we must insert $\ell$ between $\ell_{r}$ and $\ell_{l}$ in $\left\langle v_{v_{i} v_{i+1}}^{v_{i+1}}\right.$ (otherwise Invariant 2 will be violated). More precisely, we insert $\ell$ immediately left of the largest (leftmost) line $\ell^{\prime}$ in the interval $\left[\ell_{r}, \ell_{l}\right]$ of $<_{v_{i} v_{i+1}}^{v_{i+1}}$ for which $\operatorname{side}\left(\ell, \ell^{\prime}, v_{i} v_{i+1}\right)=$ right, see Figure 3b. If $\ell_{r}\left(\ell_{l}\right)$ does not exist we symbolically assign $\ell_{r}=-\infty\left(\ell_{l}=\infty\right)$ so that the interval $\left[\ell_{r}, \ell_{l}\right]$ may become unbounded. The position of $\ell$ is determined as before. If there is no line $\ell^{\prime}$ then $\ell$ becomes the rightmost line in $\left\langle v_{i} v_{i+1}\right.$.

We claim that in the assigned position $\ell$ crosses only lines that were to its left and tend to the right or lines that were to its right and tend to the leftcrossings that are unavoidable. Assume to the contrary that $\ell$ crosses a line $\hat{\ell}$ that was to its left and also tends to the left. Since we insert $\ell$ immediately to the left of $\ell^{\prime}$, the two lines $\hat{\ell}$ and $\ell^{\prime}$ also cross each other. This is a contradiction to Invariant 2 for the previous partial layout, though, since $\hat{\ell}$ crosses $\ell^{\prime}$ from left to right but eventually needs to cross $\ell^{\prime}$ again from right to left in order to reach its leftward destination. If there is no line $\ell^{\prime}$ then $\ell$ is the rightmost line in $\left\langle v_{i} v_{i+1} v_{i+1}\right.$ by definition and cannot cross $\hat{\ell}$. Similarly, assume that $\ell$ crosses 
a line $\tilde{\ell}$ that was to its right and also tends to the right. Then $\ell_{l}<v_{i} v_{i+1} v_{i} \tilde{\ell}$ since otherwise we would have placed $\ell$ left of $\tilde{\ell}$ in the interval $\left[\ell_{r}, \ell_{l}\right]$. But this means that $\tilde{\ell}$ crosses $\ell_{l}$ from right to left, which again violates Invariant 2 for the previous partial layout: there must be a second crossing, where $\tilde{\ell}$ crosses $\ell_{l}$ from left to right in order to reach its rightward destination. If $\ell_{l}=\infty$ we would have placed $\ell$ left of $\tilde{\ell}$ which is also a contradiction. So Invariant 2 holds for the selected position of $\ell$.

Finally, we show that Invariant 1 holds for the position of $\ell$ in $<_{v_{i} v_{i+1}}^{v_{i+1}}$. The first possibility for a violation is a line $\hat{\ell}$ with $\operatorname{side}\left(\ell, \hat{\ell}, v_{i} v_{i+1}\right)=$ left that is still to the right of $\ell$ but does not continue further along $v_{i+1} v_{i+2}$. By definition $\hat{\ell}$ can only be right of $\ell$ if $\hat{\ell}<v_{i} v_{i+1} v_{i+1} \ell^{\prime}$. But then Invariant 1 would have been violated before by $\hat{\ell}$ and $\ell^{\prime}$. The other possibility for a violation of Invariant 1 is a line $\tilde{\ell}$ with $\operatorname{side}\left(\ell, \tilde{\ell}, v_{i} v_{i+1}\right)=$ right that is still to the left of $\ell$ but does not continue further along $v_{i+1} v_{i+2}$. By definition this can only be the case if $\ell_{l}<v_{i} v_{i+1} \tilde{\ell}$. But this means that Invariant 1 would have been violated before by $\tilde{\ell}$ and $\ell^{\prime}$.

Since both invariants hold at the end of Algorithm 11 we have proven its correctness. By Invariant 1 all vertices are admissible, and by Invariant 2 the final line layout realizes exactly the unavoidable crossings and is thus crossingminimal by Lemma 2 The running time of Phase 1 is $O\left(|\mathcal{L}| \cdot N_{\mathcal{L}}\right)$. The running time of Phase 2 is again $O\left(|\mathcal{L}| \cdot N_{\mathcal{L}}\right)$ since there are $2 N_{\mathcal{L}}$ insertion operations, each of which determines a position for the current line by scanning the line orders of size $O(|\mathcal{L}|)$ of the current edge.

We note that the size of a solution for MLCM-PA is $\Omega\left(N_{\mathcal{L}}\right)$ and thus the running time of our algorithm is only a factor of $|\mathcal{L}|$ away from the output size. Since for the total edge size $N_{\mathcal{L}}$ we have $N_{\mathcal{L}} \in O(|\mathcal{L}| \cdot|V|)$, the running time of Algorithm 1 can also be expressed as $O\left(|\mathcal{L}|^{2} \cdot|V|\right)$.

By Lemma 1, we can reduce any instance of MLCM-T1 to an equivalent instance of MLCM-PA in linear time. We thus obtain the following corollary.

Corollary 1. Given a metro graph $(G, \mathcal{L})$ in which the degree of any terminus $v$ of any line in $\mathcal{L}$ equals 1 , we can use Algorithm 1 to compute a crossing-minimal line layout in $O\left(|\mathcal{L}| \cdot N_{\mathcal{L}}\right)$ time.

\section{Conclusions}

In this paper we have presented a new algorithm that improves the best previous algorithms for both the MLCM-PA and the MLCM-T1 problem. The running time of the new algorithm is $O\left(|\mathcal{L}| \cdot N_{\mathcal{L}}\right)$, where $N_{\mathcal{L}} \in O(|\mathcal{L}| \cdot|V|)$.

We conclude with two observations about practical MLCM instances as found, for example, in Ovenden's book [3. First, the number of lines $|\mathcal{L}|$ in a transport network is usually much smaller than the size of the underlying graph $G$. Since the output size is already $\Omega\left(N_{\mathcal{L}}\right)$, our algorithm runs in linear time if the number of lines is constant. Second, many lines in practice indeed terminate at degree-1 vertices of the underlying graph as modeled in the MLCM-T1 variant. Still, most 
networks also have some lines that start or end in non-leaf vertices. We therefore suggest to use the ILP formulation of Asquith et al. [1] (or a simple exhaustivesearch algorithm) to determine an optimal terminus side assignment for those lines. We can then transform the original MLCM-P instance together with the additional terminus side assignments into an MLCM-PA instance that can be solved efficiently with our algorithm.

There are a few remaining open problems in MLCM. First of all, it is still an unsolved question whether the general MLCM problem (without periphery condition) is NP-hard for general plane graphs or even for paths. Another interesting open question is whether the NP-hard problem MLCM-P is fixed-parameter tractable for a suitable small parameter, such as the maximum multiplicity of the edges. Furthermore, no approximation algorithms for MLCM-P are known so far.

Acknowledgments. We thank Joachim Gudmundsson, Damian Merrick, and Thomas Wolle for initial discussions about the problem during a visit in Sydney.

\section{References}

1. Asquith, M., Gudmundsson, J., Merrick, D.: An ILP for the metro-line crossing problem. In: Harland, J., Manyem, P. (eds.) Proc. 14th Computing: The Australasian Theory Symp. (CATS 2008). CRPIT, vol. 77, pp. 49-56. Australian Comput. Soc. (2008)

2. Argyriou, E., Bekos, M.A., Kaufmann, M., Symvonis, A.: Two polynomial time algorithms for the metro-line crossing minimization problem. In: Tollis, I.G., Patrignani, M. (eds.) GD 2008. LNCS, vol. 5417, pp. 336-347. Springer, Heidelberg (2009)

3. Ovenden, M.: Metro Maps of the World. Capital Transport Publishing (2003)

4. Stott, J.M., Rodgers, P.: Metro map layout using multicriteria optimization. In: Proc. 8th Internat. Conf. Information Visualisation (IV 2004), pp. 355-362. IEEE, Los Alamitos (2004)

5. Hong, S.H., Merrick, D., do Nascimento, H.A.D.: Automatic visualization of metro maps. J. Visual Languages and Computing 17, 203-224 (2006)

6. Nöllenburg, M., Wolff, A.: A mixed-integer program for drawing high-quality metro maps. In: Healy, P., Nikolov, N.S. (eds.) GD 2005. LNCS, vol. 3843, pp. 321-333. Springer, Heidelberg (2006)

7. Hahn, W.C., Weinberg, R.A.: A subway map of cancer pathways (2002); Poster in Nature Reviews Cancer

8. Benkert, M., Nöllenburg, M., Uno, T., Wolff, A.: Minimizing intra-edge crossings in wiring diagrams and public transportation maps. In: Kaufmann, M., Wagner, D. (eds.) GD 2006. LNCS, vol. 4372, pp. 270-281. Springer, Heidelberg (2007)

9. Bekos, M.A., Kaufmann, M., Potika, K., Symvonis, A.: Line crossing minimization on metro maps. In: Hong, S.-H., Nishizeki, T., Quan, W. (eds.) GD 2007. LNCS, vol. 4875, pp. 231-242. Springer, Heidelberg (2008) 